\title{
Adaptation scolaire et sociale d'élèves ayant reçu des services éducatifs complémentaires pour troubles de comportement dès le début de leur scolarité
}

\author{
Michèle Déry, Myriam Laventure, Jean Toupin, Pierrette Verlaan et Robert \\ Pauzé
}

Volume 10, numéro 1, 2007

Apprendre et former : pour quelles réussites scolaires?

URI : https://id.erudit.org/iderudit/1016857ar

DOI : https://doi.org/10.7202/1016857ar

Aller au sommaire du numéro

Éditeur(s)

Faculté d'éducation, Université de Sherbrooke

ISSN

1911-8805 (numérique)

Découvrir la revue

Citer cet article

Déry, M., Laventure, M., Toupin, J., Verlaan, P. \& Pauzé, R. (2007). Adaptation scolaire et sociale d'élèves ayant reçu des services éducatifs complémentaires pour troubles de comportement dès le début de leur scolarité. Nouveaux cahiers de la recherche en éducation, 10(1), 47-62.

https://doi.org/10.7202/1016857ar
Résumé de l'article

L'étude examine l'évolution d'un groupe d'élèves suivi à l'école pour des troubles de comportement dès le $1^{\mathrm{er}}$ cycle du primaire et cherche à déterminer si leur adaptation scolaire et sociale au $3^{\mathrm{e}}$ cycle devient comparable à celle d'enfants n'ayant pas eu de troubles de comportement en début de scolarité. Les résultats montrent que les troubles de comportement sont relativement stables en dépit des services reçus. Cependant, l'adaptation sociale des élèves est assez comparable à celle du groupe témoin pour ce qui est de la délinquance et de la consommation de psychotropes. Leur rendement scolaire demeure toutefois faible malgré de bonnes habiletés cognitives. Ces résultats sont discutés dans une perspective d'intervention. 


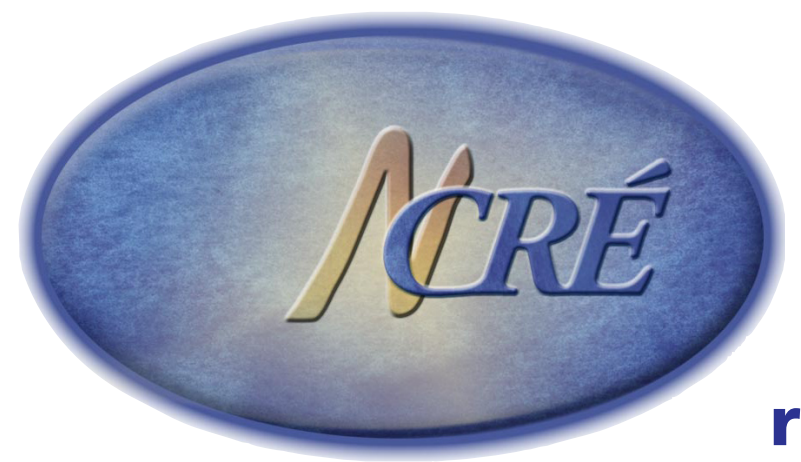

\title{
Adaptation scolaire et sociale d'élèves ayant
} reçu des services éducatifs complémentaires pour troubles de comportement dès le début de leur scolarité

\author{
Michèle Déry, Myriam Laventure, Jean Toupin, \\ Pierrette Verlaan et Robert Pauzé \\ Université de Sherbrooke
}

Résumé - L'étude examine l'évolution d'un groupe d'élèves suivi à l'école pour des troubles de comportement dès le $1^{\text {er }}$ cycle du primaire et cherche à déterminer si leur adaptation scolaire et sociale au $3^{\mathrm{e}}$ cycle devient comparable à celle d'enfants n'ayant pas eu de troubles de comportement en début de scolarité. Les résultats montrent que les troubles de comportement sont relativement stables en dépit des services reçus. Cependant, l'adaptation sociale des élèves est assez comparable à celle du groupe témoin pour ce qui est de la délinquance et de la consommation de psychotropes. Leur rendement scolaire demeure toutefois faible malgré de bonnes habiletés cognitives. Ces résultats sont discutés dans une perspective d'intervention.

Abstract - The research reported on here examined the progress of a group of pupils who were followed in school from Cycle 1 of elementary school. The goal was to determine if these pupils' academic and social adjustment when they reached Cycle 3 was comparable to that of children who had no behavioural disorders when they started school. Our findings show that behavioural disorders are comparatively stable regardless of services received. On the other hand, these pupils' social adjustment was reasonably comparable to that of the control group when it came to delinquency and the consumption of psychotropic drugs. Their academic performance, however, remained weak even when they had good cognitive skills. Our findings are discussed from the point of view of intervention. 


\section{Problématique}

Les troubles de comportement chez les élèves du primaire font l'objet d'une préoccupation accrue au Québec, non seulement en raison de la prévalence élevée de ces troubles, mais aussi de l'augmentation du nombre d'enfants recevant des services éducatifs complémentaires à l'école pour ce type de difficultés (Conseil supérieur de l'éducation, 2001). Le pourcentage d'élèves qui y sont suivis pour troubles de comportement a, en effet, triplé au cours des deux dernières décennies, passant de $0,78 \%$ en 1985 pour s'établir à 2,5\% en 2000 (Ibid.).

La nécessité d'intervenir tôt auprès des élèves qui ont des troubles de comportement suscite un large consensus (Gagnon et Vitaro, 2003). C'est ainsi qu'à l'école, les élèves identifiés avec ces troubles bénéficient de services éducatifs complémentaires prenant habituellement la forme d'un accompagnement par un professionnel non enseignant (psychoéducateur, psychologue, éducateur spécialisé, etc.) qui œuvre avec l'élève à trouver des solutions à ses difficultés (Gouvernement du Québec, 2002; Trépanier et Dessureault, 2006). Or, de manière surprenante, il existe peu d'informations sur l'évolution des élèves qui ont reçu ces services complémentaires. Disposer d'informations sur l'adaptation scolaire et sociale ultérieure de ces enfants est pourtant essentiel pour déterminer si les services habituellement mis en place à l'école répondent à leurs différents besoins ou doivent être ajustés pour qu'ils facilitent leur réussite scolaire (Frick, 2004). Les besoins de ces enfants sont, toutefois, très grands et représentent un véritable défi pour les intervenants du milieu scolaire tant en raison de la nature des difficultés comportementales présentées que des problèmes scolaires et sociaux qui leur sont associés (Vernberg, Roberts, Randall, Biggs, Nyre et Jacobs, 2006).

Les troubles précoces de comportement sont, en effet, reconnus pour leur sévérité et leur risque élevé de persistance dans le temps (American Psychiatric Asssociation, 2000), tout comme pour leur résistance aux traitements (Kazdin et Wassell, 2000; Vernberg et al., 2006). Les enfants identifiés avec ces troubles au primaire le sont surtout en raison de leurs conduites antisociales et perturbatrices, incluant les agressions verbales et physiques, l'intimidation, l'impulsivité/hyperactivité, la désobéissance et le refus d'encadrement (Fortin et Strayer, 2000). Dans la majorité des cas, toutefois, la fréquence et la récurrence de ces conduites sont telles qu'elles rencontrent les critères diagnostiques de différents troubles de la santé mentale (Déry, Toupin, Pauzé et Verlaan, 2004; Kershaw et Sonuga-Barke, 1998; Mattison, Gadow, Sprafkin et Nolan, 2002 ; Nelson, Babyak, Gonzalez et Benner, 2003; Place, Wilson, Martin et Hulsmeier, 2000). C'est ainsi que près des deux tiers des élèves suivis pour troubles de comportement au primaire répondent aux critères d'un trouble de l'opposition avec provocation, voire d'un trouble des conduites ${ }^{1}$, ces deux troubles de nature antisociale s'observant le plus souvent en cooccurrence d'un trouble déficitaire de l'attention/hyperactivité (TDA/H) (Déry et al., 2004; Kershaw et Sonuga-Barke, 1998; Mattison et al., 2002; Place et al., 2000). Soulignons que la cooccurrence

1 Le trouble de l'opposition avec provocation et le trouble des conduites sont décrits dans le Diagnostic and Statistical Manual of Mental Disorders (American Psychiatric Association, 2000). Le premier fait référence à une façon d'être négative et hostile chez l'élève, caractérisée par l'argumentation, le refus d'obéir aux règles, l'irritabilité et les crises de colère. Le second consiste en un ensemble de conduites répétitives et persistantes d'agression envers les autres, de destruction de biens matériels, de mensonges, de vols ou de violation des règles établies. Le trouble des conduites est plus fréquent à l'adolescence qu'à l'enfance et constitue un problème particulièrement grave chez les jeunes enfants (Breton, Bergeron, Valla, Berthiaume, Gaudet, Lambert, St-Georges, Houde et Lépine, 1999). 
du TDA/H et d'un trouble oppositionnel ou des conduites chez les enfants est associée aux problèmes comportementaux les plus agressifs et les plus persistants (Waschbusch, 2002).

Bien que les troubles précoces de comportement représentent déjà un défi de taille, le travail d'intervention se complexifie du fait que les élèves qui présentent ces troubles sont à risque élevé d'avoir ou de développer d'importants problèmes scolaires et sociaux (Fortin et Strayer, 2000). Sur le plan scolaire, ces enfants sont nombreux à éprouver des difficultés d'apprentissage ou à cumuler des retards; en outre, une portion élevée fréquente la classe spéciale au primaire (Déry, Toupin, Pauzé et Verlaan, 2005 ; Gouvernement du Québec, 1999). Des études appuient aussi l'idée que les enfants qui ont des troubles précoces de comportement peuvent avoir de moins bonnes habiletés cognitives que les élèves qui n'ont pas ces troubles (Moffitt et Caspi, 2006; Toupin, Déry, Pauzé, Mercier et Fortin, 2000), une différence constatable au-delà du rôle éventuellement joué par le statut socioéconomique, la motivation des enfants ou leur manque de contrôle (Lynam, Moffitt et Stouthamer-Loeber, 1993). Enfin, les troubles précoces de comportement sont reconnus comme d'importants prédicteurs du décrochage scolaire à l'adolescence (Battin-Pearson, Newcomb, Abbott, Hill, Catalano et Hawkins, 2000 ; Fortin, Royer, Potvin, Marcotte et Yergeau, 2004) et, en corollaire, sont associés à de faibles taux d'obtention de diplômes et d'intégration sur le marché du travail (Beekhoven et Dekkers, 2005).

Sur le plan de l'adaptation sociale, outre le fait qu'ils se caractérisent souvent par des conduites agressives envers les autres, les troubles précoces de comportement sont associés au développement d'un mode de vie plus déviant, exposant les enfants à d'autres facteurs de risque. C'est particulièrement le cas de la délinquance et de la consommation de substances psychotropes qui, d'après Moffitt et Caspi (2006), peuvent «piéger» les enfants et compromettre leurs chances d'adaptation ultérieure. Les troubles de comportement ont, en effet, été reliés au rejet des élèves qui ont une bonne adaptation sociale et à l'affiliation à des pairs délinquants (Fortin et Strayer, 2000). S'il n'est pas clair que ces facteurs contribuent à l'émergence des troubles ou en constituent des conséquences (Alvarez et Ollendick, 2003; Vitaro, Brendgen, Pagani, Tremblay, McDuff, 1999), les résultats des études suggèrent néanmoins un réseau social de plus faible densité et de moindre qualité chez les enfants qui ont des difficultés comportementales, ce qui peut contribuer à les maintenir sur la voie de la déviance. Ces enfants sont aussi à risque élevé de s'initier précocement à la consommation d'alcool et de drogues (Hinshaw et Lee, 2003 ; Laventure, Déry et Pauzé, 2006a), ce qui peut augmenter le risque de développer des problèmes de consommation (abus, dépendance) à l'adolescence (Kuperman, Chan, Kramer et Bierut, 2005; Lambert, 2005).

L'étude présentée dans cet article ${ }^{2}$ porte sur l'évolution d'un groupe d'élèves qui, dès le premier cycle du primaire, ont reçu des services d'accompagnement de type psychoéducatif à l'école pour des troubles de comportement (TC). Le premier objectif de l'étude est d'examiner si quatre ans plus tard, soit au troisième et dernier cycle du primaire, les troubles de ces élèves ont diminué d'intensité ou se sont résorbés. Un second objectif est de déterminer si, au troisième cycle, l'adaptation scolaire et sociale de ces élèves est comparable à celle d'un groupe témoin, composé d'élèves qui, au début de leur scolarité, ne manifestaient pas de troubles de comportement ni ne recevaient de services éducatifs complémentaires pour ce type de difficultés.

2 Cette étude a bénéficié de l'appui financier du Conseil de recherche en sciences humaines du Canada (CRSH), du Fonds québécois pour la recherche sur la société et la culture (FQRSC) et de l'Université de Sherbrooke. Les auteurs tiennent à remercier les parents, les enfants et les enseignants qui ont participé à la recherche et expriment leur gratitude aux intervenants psychosociaux pour leur collaboration remarquable. 


\section{Méthode}

\subsection{Sélection des participants}

Les élèves qui participent à l'étude constituent un sous-échantillon recruté dans le cadre d'une recherche longitudinale plus vaste portant sur les facteurs de persistance des troubles de comportement et les parcours dans les services scolaires et sociaux. De l'échantillon de cette recherche, la présente étude ne retient que les enfants qui étaient au premier cycle du primaire au début de la recherche et qui, quatre ans plus tard, sont toujours inscrits à l'école primaire.

\subsection{1 Élèves présentant des troubles du comportement}

Les élèves présentant des troubles de comportement proviennent de trois commissions scolaires situées en Estrie et en Montérégie. Tous les élèves des écoles primaires participantes (de la $1^{\text {re }}$ à la $6^{\mathrm{e}}$ année) qui recevaient des services complémentaires pour troubles de comportement ont été ciblés, à l'exclusion de ceux qui avaient aussi une déficience intellectuelle ou sensorielle ou qui ne vivaient pas avec au moins un de leurs parents biologiques. Les parents de ces élèves ont d'abord été joints par les intervenants psychosociaux œuvrant dans les écoles afin qu'ils consentent à participer à la recherche. Un accord a été donné pour 324 élèves (dont 88 enfants de premier cycle), ce qui représente un taux de participation de $62,3 \%$ par rapport aux enfants ciblés.

L'échantillon recruté s'est avéré proportionnellement comparable à l'échantillon initial sur la provenance des élèves, leur répartition dans chaque ordre scolaire, le ratio garçons-filles et le taux d'élèves scolarisés en classe spéciale. Des 88 élèves recrutés au premier cycle, 57 enfants (soit $65 \%$ ) ont pu être réévalués quatre ans plus tard alors qu'ils étaient au troisième cycle du primaire. Bien qu'importante, l'attrition s'est avérée aléatoire, les caractéristiques des non-participants ne se différenciant pas significativement de celles des participants au début de l'étude.

\subsection{2 Élèves du groupe témoin}

Le groupe témoin a été recruté dans les écoles primaires des secteurs ouvriers desservis par une commission scolaire de l'Estrie. Ces secteurs ont surtout été ciblés pour réduire les écarts socioéconomiques entre les élèves avec ou sans troubles de comportement. Des élèves ont d'abord été sollicités au hasard parmi les élèves de la $1^{\text {re }}$ à la $6^{\mathrm{e}}$ année. Les parents de ces élèves ont été joints par lettre pour les informer des objectifs de l'étude et pour leur demander s'ils désiraient y participer. Cette procédure a été répétée jusqu'à l'obtention d'un accord de participation pour environ 150 élèves. Nous nous sommes ensuite assurés que ces élèves n'avaient pas reçu de services éducatifs spécialisés pour difficultés comportementales ou émotionnelles durant l'année scolaire en cours ou durant l'année précédente. Puis, à partir de cet échantillon, nous avons constitué un groupe témoin proportionnellement apparié à l'âge, au sexe et au statut socioéconomique des enfants présentant des troubles du comportement. Ce groupe témoin comptait 56 élèves de premier cycle. Quatre ans plus tard, 42 des 56 élèves ( $75 \%)$ ont pu être réévalués. Encore ici, la perte de sujets dans le groupe témoin entre les deux temps de mesure 
apparaît aléatoire, les participants ne se distinguant pas des non-participants sur les variables mesurées initialement.

Les élèves du groupe témoin $(\mathrm{N}=42)$ et ceux du groupe suivi pour troubles de comportement (groupe $\mathrm{TC}, \mathrm{N}=57$ ) qui participent à l'étude ne se distinguaient pas significativement au premier cycle du primaire sur l'âge moyen [7,6 ans vs 7,4 ans, $\mathrm{t}(97)=0,99$, n.s.], la proportion de filles $[38,1 \%$ vs $21,1 \%$, c2 $(1,99)=2,67]$ et le statut socioéconomique [indice de 5,1 vs indice $5,5, \mathrm{t}(97)=-2,05$, n.s. $]^{3}$.

\subsection{Variables mesurées}

\subsubsection{Troubles de comportement}

L'information sur la nature et la sévérité des troubles de comportement des élèves du groupe TC a été recueillie au début de l'étude puis quatre ans plus tard auprès de deux informateurs: le parent (celui qui s'occupe le plus des soins et de l'éducation de l'enfant) et l'enseignant. ${ }^{4} \mathrm{La}$ version française du Diagnostic Interview Schedule for Children - Revised (Breton, Bergeron, Valla, Berthiaume et St-Georges, 1998) a été utilisée à cette fin. Il s'agit d'un questionnaire de type «oui-non», développé à partir des critères du Diagnostic and Statistical Manual of Mental Disorders (DSM-III-R) (American Psychiatric Association, 1987), permettant d'établir les symptômes et la présence des troubles de la santé mentale les plus fréquemment rencontrés chez les enfants. Les parents et les enseignants ont complété les sections se rapportant aux symptômes de trouble de l'opposition avec provocation, de trouble des conduites et de TDA/H que les enfants ont pu manifester au cours des six mois précédant la passation du questionnaire.

Nous avons légèrement modifié le questionnaire pour qu'il englobe, aussi, les critères plus récents du Diagnostic and Statistical Manual of Mental Disorders (DSM-IV-TR) (American Psychiatric Association, 2000). Les valeurs du Kappa calculées pour évaluer la correspondance des versions DSM-III-R ou DSM-IV-TR du questionnaire pour établir la présence/absence des troubles chez l'enfant se sont avérées bonnes, et ce, tant lorsque l'informateur est l'enseignant (trouble de l'opposition: 0,87 ; trouble des conduites : 0,$90 ; \mathrm{TDA} / \mathrm{H}: 0,67$ ) ou le parent (trouble de l'opposition: 0,88; trouble des conduites: 0,65 ; TDA/H: 0,73) (Déry et al., 2004). Dans l'étude, la présence d'un trouble extériorisé a été considérée lorsque l'ensemble des symptômes requis pour le diagnostic a été rapporté par le parent ou par l'enseignant. Les nombres minimum de symptômes requis pour le trouble oppositionnel et pour le trouble des conduites sont respectivement de quatre et de trois symptômes. Pour le TDA/H, l'enfant doit manifester au moins six symptômes d'inattention ou au moins six symptômes d'hyperactivité/impulsivité (ou six symptômes dans chacune de ces deux dimensions) pour que le diagnostic soit considéré.

3 Le statut socioéconomique est estimé à partir d'un indice en huit points calculé à partir du revenu familial, de la scolarité et du statut d'emploi des parents (Toupin, 1993).

4 Plusieurs auteurs recommandent de considérer plus d'une source d'information pour établir la présence de troubles de comportement puisque ces sources peuvent s'avérer complémentaires (Breton et al., 1999; Jensen, Rubio-Stipec, Canino, Bird, Dulcan et Schwab-Stone, 1999; Mitsis, McKay, Schulz, Newcorn et Halperin, 2000). Les parents et les enseignants sont néanmoins considérés comme des informateurs plus valides que l'enfant pour établir la présence des troubles extériorisés (Breton et al., 1999; Jensen et al., 1999; Mitsis et al., 2000). 


\subsubsection{Adaptation scolaire}

Cinq indicateurs ont été utilisés pour estimer l'adaptation scolaire des élèves au troisième cycle du primaire. Il s'agit du rendement scolaire en français et en mathématiques, des habiletés cognitives, du placement scolaire et des services psychoéducatifs éventuellement reçus.

Rendement scolaire en français et en mathématiques. Le rendement scolaire de l'élève a été estimé par le parent (groupe témoin) et l'enseignant (groupe TC) à l'aide d'une échelle figurant dans le Child Behavior Checklist (Achenbach et Edelbrock, 1983). Cette échelle en cinq points porte sur les résultats en français et en mathématiques. Elle a été dichotomisée de manière à regrouper, d'une part, les choix «très en dessous de la moyenne» et «en dessous de la moyenne» et, d'autre part, les choix «dans la moyenne», «au-dessus de la moyenne» et «très au-dessus de la moyenne». L'échelle reflète, ainsi, l'absence ou la présence de difficultés dans ces deux matières scolaires.

Habiletés cognitives. L'Échelle de vocabulaire en images Peabody (Dunn, Thériault-Whalen et Dunn, 1993) a été utilisée auprès des élèves pour estimer leurs habiletés cognitives. Il s'agit d'un test de vocabulaire réceptif validé au Canada français. L'échelle permet le calcul d'un score standardisé qui peut être employé comme indicateur des habiletés cognitives des enfants en raison des corrélations relativement élevées que ce score entretient avec les échelles d'intelligence Stanford-Binet et Wechsler pour enfants (corrélations médianes entre 0,62 et 0,66) (Dunn et al., 1993).

Placement scolaire et services scolaires complémentaires. Un questionnaire issu de l'Enquête québécoise sur la santé mentale des jeunes (Valla, Breton, Bergeron, Gaudet, Berthiaume, St-Georges, Daveluy, Tremblay, Lambert, Houde et Lépine, 1994) a été utilisé auprès du parent pour savoir si l'enfant avait reçu à l'école des services psychoéducatifs (ou psychologiques) durant l'année scolaire et s'il avait fréquenté la classe spéciale au cours de cette période.

\subsubsection{Adaptation sociale}

Afin d'estimer l'adaptation sociale des élèves au troisième cycle du primaire, cinq autres indicateurs ont été mesurés, incluant la qualité de leur insertion sociale ainsi que leurs conduites déviantes (agression verbale, agression physique, délinquance, consommation de psychotropes).

Insertion sociale de l'enfant. Adapté de l'Échelle de compétence sociale du Child Behavior Checklist pour l'Enquête québécoise sur la santé mentale des jeunes (Valla et al., 1994), 1'instrument a été utilisé auprès du parent pour estimer la qualité de l'insertion sociale de l'enfant. Il permet le calcul d'un score global à partir de variables telles que le nombre d'organisations sociales et sportives dont fait partie l'enfant, le nombre d'amis proches formant son réseau social et le nombre d'activités sociales auxquelles il participe. La cohérence interne et la fidélité test-retest de l'échelle sont excellentes, étant respectivement de 0,99 et 0,97. Un score élevé à l'échelle reflète une bonne insertion sociale.

Conduites agressives. L'information sur les conduites d'agression de l'enfant a été recueillie auprès du parent à l'aide des Échelles d'agression de Bjorkqvist, Lagerspetz et Österman (1992). Ces échelles permettent le calcul de trois scores relatifs à l'agression physique, à l'agression 
verbale et à l'agression indirecte. La cohérence interne des échelles est élevée, allant de 0,89 à 0,96 . Seuls les scores se rapportant à l'agression physique et verbale sont utilisés ici.

Conduites délinquantes. Tirées de l'instrument utilisé pour évaluer les troubles du comportement présenté précédemment, les conduites délinquantes de l'enfant au cours des douze derniers mois ont été rapportées par les parents à partir de questions de type «oui-non». Ces questions se rapportaient principalement aux vols (avec ou sans agression) et aux actes de vandalisme. Le pourcentage d'enfants ayant commis au moins un de ces actes délictueux est la variable à l'étude.

Consommation de psychotropes. Deux instruments ont été utilisés auprès des élèves en fonction de leur âge. Pour les 11 ans et moins, le questionnaire employé est celui conçu pour l'Enquête longitudinale nationale sur les enfants et les jeunes (Statistique Canada, 1996). Cet outil comporte 12 questions se rapportant, entre autres, à leur consommation de cigarettes, d'alcool et de drogues. Pour les élèves de 12 ans et plus, c'est la Grille de dépistage de consommation problématique d'alcool et de drogues (Germain, Guyon, Landry, Tremblay, Brunelle, et Bergeron, 2005) qui a été utilisée. Cet outil est plus élaboré que celui passé aux enfants plus jeunes, mais permet aussi d'obtenir les mêmes informations de base se rapportant au fait d'avoir déjà consommé différents psychotropes (alcool, cannabis, cigarette, etc.). Les données sur la consommation de psychotropes n'ont été recueillies qu'auprès de l'élève; cette consommation se faisant le plus souvent à l'insu des parents, les données recueillies auprès de l'enfant sont reconnues comme les plus fiables et les plus rigoureuses (Williams et Nowatzki, 2005).

\subsection{Déroulement}

Tous les élèves formant l'échantillon (groupe TC et groupe témoin) et un de leurs parents ont été rencontrés séparément à domicile par des assistants de recherche spécifiquement formés à la passation des questionnaires. Les enseignants des élèves du groupe TC ont, quant à eux, répondu au questionnaire sur les troubles de comportement des élèves par téléphone. Il est à noter que les informations en provenance des enseignants aux deux temps de mesure (premier cycle et troisième cycle) n'ont pu être obtenues que pour 44 des 57 élèves du groupe TC. Les enseignants des élèves du groupe témoin n'ont pas été joints au deuxième temps de mesure.

Préalablement à la passation des questionnaires, les parents ont signé un formulaire de consentement les informant des objectifs de l'étude et des risques et bienfaits reliés à leur participation. Les parents des élèves du groupe TC donnaient également leur consentement pour que nous contactions l'enseignant de leur enfant. À la fin de la rencontre, chaque participant (parent, enfant, enseignant) recevait une compensation monétaire pour le temps de participation à l'étude.

\subsection{Analyses}

Afin d'établir si les troubles de comportement des élèves du groupe TC s'étaient atténués ou résorbés entre le premier cycle et le troisième cycle du primaire (objectif 1), nous avons choisi de recourir à des statistiques descriptives (fréquence des troubles selon qu'il s'agisse du trouble oppositionnel, du trouble des conduites ou du TDA/H) et à des tests-t pour échantillons 
dépendants sur les symptômes de chacun de ces troubles puisque les élèves du groupe TC ne pouvaient être comparés qu'à eux-mêmes dans le temps.

Soulignons que le nombre relativement faible de participants dans ce groupe rendait précaire l'utilisation d'analyses statistiques plus complexes, telles l'analyse de trajectoire latente, pour rendre compte de l'évolution des symptômes des élèves (Muthén et Curran, 1997). Les analyses unidimensionnelles retenues ici ont toutefois l'avantage de permettre une description détaillée des différentes difficultés comportementales de ces élèves.

Des analyses statistiques unidimensionnelles (test-t et khi-carré) ont aussi été retenues afin de décrire et comparer, au troisième cycle, les élèves des groupes TC et témoin sur les différents indicateurs de l'adaptation scolaire et sociale utilisés dans l'étude (objectif 2). Ces analyses unidimensionnelles ont été suivies d'une analyse de régression logistique intégrant tous ces indicateurs. Cette dernière a été réalisée afin de tenir compte des corrélations entre les différents indicateurs et de déterminer ceux qui permettaient le mieux de rendre compte de l'appartenance aux groupes TC ou témoin. La méthode de sélection des indicateurs qui a été retenue pour cette analyse de régression est la méthode "par étape». Bien que controversée pour vérifier un modèle théorique, cette méthode s'avère particulièrement appropriée lorsque le but de l'analyse est uniquement l'identification des prédicteurs d'un phénomène à des fins pratiques (Menard, 2001), comme c'est le cas ici.

\section{Résultats}

Le tableau 1 montre les troubles de comportement présentés par les élèves du groupe TC au premier cycle du primaire puis, quatre ans plus tard, au troisième cycle. Rappelons que des données sur ces troubles provenant à la fois des parents et des enseignants ne sont disponibles que pour 44 élèves du groupe TC. Comme on peut le constater, au premier cycle, les difficultés comportementales des élèves répondaient aux critères diagnostiques d'au moins un trouble extériorisé (normes du DSM-IV-TR) dans 84,1 \% des cas. Quatre années plus tard, près de trois enfants sur quatre présentent encore au moins un trouble extériorisé, toujours selon les critères du DSM-IV-TR. Le diagnostic le plus fréquemment rapporté aux deux temps de mesure est le TDA $/ \mathrm{H}$, mais plus de $60 \%$ des élèves du groupe TC présentent à la fois ce trouble et un trouble oppositionnel ou un trouble des conduites (pourcentage non présenté ici). Si le nombre d'enfants présentant toujours un trouble au troisième cycle du primaire demeure élevé, les tests-t pour échantillons dépendants révèlent que le nombre moyen de symptômes de TDA/H et de trouble oppositionnel a diminué de manière significative entre les deux temps de mesure. Cependant, l'évolution du nombre de symptômes de trouble des conduites entre le premier cycle et le troisième cycle du primaire a été très variable d'un élève à l'autre et ne s'est pas avérée statistiquement significative ${ }^{5}$.

5 Les conclusions sont les mêmes lorsque des tests non paramétriques sont utilisés pour comparer l'évolution des symptômes de ces troubles entre les deux temps de mesure. 
Tableau 1

Troubles de comportement présentés par les élèves du groupe $\mathrm{TC}$ au $1^{\mathrm{er}}$ et $3^{\mathrm{e}}$ cycles du primaire $(\mathrm{N}=44)$ selon l'enseignant ou le parent

\begin{tabular}{|l|l|l|l|l|}
\hline \multicolumn{1}{|c|}{ Troubles de comportement } & \multicolumn{1}{c|}{$\mathbf{1}^{\text {er }}$ cycle } & \multicolumn{1}{c|}{$\mathbf{3}^{\text {e }}$ cycle } & (43) \\
\hline Diagnostic & $\%$ & 77,3 & 63,6 & \\
\hline - TDA/H & $\%$ & 55,5 & 45,5 & \\
\hline - Trouble oppositionnel & $\%$ & 40,9 & 25,0 & \\
\hline - Trouble des conduites & $\%$ & 84,1 & 72,7 & \\
\hline - Au moins un trouble & $\%$ & $13,7(4,3)$ & $10,7(3,9)$ & $4,14^{* * *}$ \\
\hline Nombre de symptômes & M (é.t.) & $4,4(2,7)$ & $3,3(2,5)$ & $2,44^{*}$ \\
\hline - TDA/H & M (é.t.) & $2,0(2,0)$ & $1,4(1,6)$ & 1,18 \\
\hline - Trouble oppositionnel & M (é.t.) &
\end{tabular}

Note: TDA/H $=$ Trouble déficitaire de l'attention avec hyperactivité.

$* \mathrm{p}<0,05 ; * * * \mathrm{p}<0,001$.

Le fait que la majorité des élèves du groupe TC présente encore au moins un trouble extériorisé au troisième cycle du primaire laisse présager une moins bonne adaptation scolaire et sociale que les élèves du groupe témoin. Le tableau 2 montre que sur le plan scolaire, près de $75 \%$ des élèves du groupe TC font toujours 1'objet d'un accompagnement psychoéducatif pour des difficultés comportementales. Bien que ce taux soit beaucoup plus élevé que celui observé dans le groupe témoin, ce sont néanmoins $14 \%$ des élèves de ce groupe qui ont reçu ce type de services à la fin du primaire, un taux non négligeable sur lequel nous reviendrons lors de la discussion des résultats. Il n'en demeure pas moins qu'au troisième cycle, les élèves du groupe TC sont trois fois plus nombreux que ceux du groupe témoin à éprouver des difficultés en français et en mathématiques, et quatre fois plus nombreux à être scolarisés dans une classe spéciale, ces différences étant hautement significatives. Toutefois, les scores moyens des deux groupes à l'échelle des habiletés cognitives demeurent statistiquement comparables. 
Tableau 2

Indicateurs de l'adaptation scolaire et sociale des élèves des groupes TC et témoin au $3^{\mathrm{e}}$ cycle du primaire

\begin{tabular}{|c|c|c|c|c|c|}
\hline \multicolumn{2}{|l|}{ Caractéristiques scolaires } & \multirow{2}{*}{$\begin{array}{l}\begin{array}{l}\text { Groupe TC } \\
(\mathrm{N}=57)\end{array} \\
107,5(141)\end{array}$} & \multirow{2}{*}{$\begin{array}{l}\text { Groupe témoin } \\
(\mathrm{N}=42)\end{array}$} & \multirow[t]{2}{*}{$\chi^{2}(1,99)$} & \multirow{2}{*}{$\begin{array}{l}\mathrm{t}(97) \\
1,64 \\
\end{array}$} \\
\hline Habiletés cognitives & M (é.t.) & & & & \\
\hline Difficultés en français & $\%$ & 68,4 & 21,4 & $19,54 * * *$ & \\
\hline Difficultés en math & $\%$ & 57,9 & 16,7 & $15,40 * * *$ & \\
\hline Classe spéciale & $\%$ & 41,1 & 9,5 & $10,44 * * *$ & \\
\hline Accompagnement psychoéducatif & $\%$ & 73,7 & 14,3 & $31,82 * * *$ & \\
\hline \multicolumn{6}{|l|}{ Caractéristiques sociales } \\
\hline Insertion sociale & M (é.t.) & $6,2(2,9)$ & $8,0(3,7)$ & & $2,74 * *$ \\
\hline Agression physique & M (é.t.) & $0,8(0,6)$ & $0,3(0,4)$ & & $4,60 * * *$ \\
\hline Agression verbale & M (é.t.) & $1,5(0,8)$ & $0,7(0,7)$ & & $4,65 * * *$ \\
\hline Conduites délinquantes & $\%$ & 15,8 & 4,8 & 1,97 & \\
\hline - Commet des vols & $\%$ & 10,5 & 4,8 & & \\
\hline - Vandalise & $\%$ & 5,3 & 0 & & \\
\hline - Entre par effraction & $\%$ & 1,8 & 0 & & \\
\hline - Met le feu & $\%$ & 1,8 & 0 & & \\
\hline Usage de psychotropes & $\%$ & 35,1 & 21,4 & 1,57 & \\
\hline - Cigarettes & $\%$ & 15,1 & 5,3 & & \\
\hline - Alcool & $\%$ & 26,4 & 15,8 & & \\
\hline - Cannabis & $\%$ & 1,9 & 0 & & \\
\hline
\end{tabular}

$* * \mathrm{p}<0,01 ; * * * \mathrm{p}<0,001$.

Sur le plan de l'adaptation sociale, le tableau 2 montre que les élèves du groupe TC ont des scores significativement plus faibles que le groupe témoin à l'échelle d'insertion sociale, une échelle qui tient compte du nombre d'organisations sociales ou sportives auxquelles appartient l'élève, du nombre d'amis proches et du nombre d'activités réalisées avec eux. Les élèves du groupe TC obtiennent, aussi, en moyenne, des scores significativement plus élevés aux échelles d'agression physique et d'agression verbale, les différences avec le groupe témoin étant supérieures à plus d'un écart type. Néanmoins, les deux groupes ne se distinguent pas significativement sur le fait d'avoir eu des conduites délinquantes en cours d'année, bien que les élèves du groupe TC soient proportionnellement plus nombreux à présenter ces conduites. Il en est de même pour l'usage de psychotropes où les différences observées avec le groupe témoin ne sont pas suffisamment grandes pour être statistiquement significatives.

Les différents indicateurs de l'adaptation scolaire et sociale ont été introduits dans un modèle de régression logistique en tant que variables indépendantes ou «prédicteurs» afin d'établir les caractéristiques scolaires et sociales les plus étroitement associées à l'appartenance au groupe TC ou au groupe témoin au troisième cycle. Il est à noter que 9 des 10 indicateurs ont été utilisés dans ce modèle; le fait d'avoir un accompagnement psychoéducatif pour des difficultés comportementales n'ayant pas été considéré dans l'analyse. Deux indicateurs sont ressortis de l'analyse de régression comme ayant une contribution significative et indépendante 
au modèle (tableau 3): la présence de difficultés en français, entrée dans l'équation à la première étape de l'analyse, et le score d'agression verbale entré à la seconde étape. Ce modèle est hautement significatif $\left(\chi^{2}=33,94 ; \mathrm{d} .1 .=2 ; \mathrm{p}<0,001\right)$, sa capacité prédictive totale étant nettement supérieure à celle attendue aléatoirement. En effet, le modèle prédit correctement l'appartenance au groupe témoin ou au groupe TC dans $81 \%$ des cas, alors qu'en raison de la proportion d'élèves dans chaque groupe, un tirage au hasard permettrait théoriquement de classer adéquatement $57 \%$ d'entre eux.

Tableau 3

Résultat de l'analyse de régression logistique $(\mathrm{N}=99)$

\begin{tabular}{|l|l|l|l|l|l|}
\hline \multicolumn{2}{|l|}{ Variables retenues dans l'équation } & $\boldsymbol{\beta}$ (e.s.) & Wald & d.l. & $\mathbf{p}$ \\
\hline Étape 1 & Difficultés en français & $2,05(0,47)$ & 18,76 & 1 & 0,000 \\
\hline Étape 2 & Difficultés en français & $1,79(0,50)$ & 12,63 & 1 & 0,000 \\
\hline & Agression verbale & $1,03(0,46)$ & 10,39 & 1 & 0,000 \\
\hline
\end{tabular}

Table de classification

\begin{tabular}{|c|c|c|c|c|}
\hline \multirow[t]{2}{*}{ Appartenance actuelle } & \multicolumn{2}{|c|}{ Appartenance prédite } & \multirow[t]{2}{*}{$\mathbf{N}$} & \multirow{2}{*}{$\begin{array}{l}\text { Prédiction correcte } \\
(\%)\end{array}$} \\
\hline & Groupe témoin & Groupe TC & & \\
\hline Groupe témoin & 32 & 10 & 42 & 76,2 \\
\hline Groupe TC & 9 & 48 & 57 & 84,2 \\
\hline Total $(\%)$ & & & & 80,8 \\
\hline
\end{tabular}

\section{Discussion}

Le premier objectif de l'étude était d'examiner l'évolution des troubles de comportement des élèves entre le premier et le troisième cycle du primaire afin de déterminer si leurs difficultés s'étaient résorbées ou, à tout le moins, si elles avaient diminué en intensité. Sur ce plan, les résultats de l'étude permettent tout d'abord de constater que les élèves suivis pour troubles de comportement au début de leur scolarité ont des difficultés particulièrement sévères, un résultat qui rejoint les observations déjà rapportées dans d'autres études (Kershaw et Sonuga-Barke, 1998; Mattison et al., 2002; Nelson et al., 2003 ; Place et al., 2000) et que nous avons décrites dans l'introduction de l'article. L'étude montre aussi qu'en dépit des services d'accompagnement psychoéducatif reçus à l'école, une forte proportion d'élèves présente toujours des troubles importants une fois au troisième cycle du primaire. Compte tenu de la précocité des troubles et de leur gravité, un tel constat n'est sans doute pas surprenant dans la mesure où ces troubles sont non seulement reconnus pour leur forte stabilité mais, aussi, en corollaire, pour leur caractère quasi réfractaire à l'intervention (Vernberg et al., 2006). Cependant, les résultats suggèrent que les troubles des élèves peuvent être moins intenses, et ce, particulièrement dans le cas du TDA/H et du trouble oppositionnel où le nombre de symptômes a sensiblement diminué entre le premier et le troisième cycle. Il n'est toutefois pas possible de conclure à une diminution des symptômes dans le cas du trouble des conduites.

Les études épidémiologiques indiquent que la prévalence du TDA/H et du trouble oppositionnel tend à diminuer entre l'enfance et l'adolescence, mais non celle du trouble des conduites 
qui, elle, tend plutôt à augmenter entre ces deux périodes (Lahey, Schwab-Stone, Goodman, Waldman, Canino, Rathouz, Miller, Dennis, Bird et Jensen, 2000; Loeber et Keenan, 1994). En ce sens, la diminution de symptômes observée entre les deux temps de mesure ne pourrait que refléter l'évolution normale des troubles de comportement. En même temps, elle peut aussi suggérer que les services éducatifs complémentaires reçus par les enfants jouent un rôle dans la non-aggravation des troubles et leur diminution en intensité. Le devis de l'étude ne nous autorise pas à aller plus loin dans l'interprétation des résultats puisqu'il ne comprend pas un groupe d'élèves ayant des troubles de comportement qui n'auraient pas reçu l'aide offerte à l'école - une alternative bien évidemment inacceptable sur le plan éthique.

La qualité de l'adaptation scolaire et sociale des élèves a toutefois pu être estimée au troisième cycle du primaire à partir de comparaisons avec des élèves qui ne présentaient pas de troubles de comportement ni ne recevaient de services éducatifs complémentaires au début de leur scolarité. Par rapport à ce second objectif, l'étude suggère que les élèves du groupe TC ont une moins bonne adaptation scolaire que leurs pairs du groupe témoin. En effet, ils sont nettement plus nombreux à éprouver des difficultés dans l'apprentissage du français et des mathématiques et le tiers d'entre eux fréquente la classe spéciale. Il ressort particulièrement de l'étude que la présence de difficultés en français pourrait être une caractéristique déterminante. Si l'écart de rendement scolaire entre les élèves des groupes TC et témoin pourrait refléter une certaine désirabilité sociale - ce rendement a été évalué par les parents dans le groupe témoin et par les enseignants dans le groupe TC -, soulignons que les difficultés d'apprentissage des élèves qui ont des troubles de comportement sont fréquemment relevées dans la documentation (Fortin et Strayer, 2000).

Toutefois, les résultats de l'étude n'appuient pas l'idée que les élèves du groupe TC ont de moins bonnes habiletés cognitives que les autres élèves (Moffitt et Caspi, 2006; Toupin et al., 2000). Ce résultat est encourageant et suggère que ces habiletés pourraient constituer une force sur laquelle les intervenants pourraient miser. Cependant, le test utilisé ne permet qu'une estimation globale de ces habiletés et peut ne pas rendre compte de problèmes ou de déficits plus fins, notamment ceux touchant les fonctions exécutives et qui sont plus étroitement associés à la présence de TDA/H et du trouble des conduites (Toupin et al., 2000).

Sur le plan de l'adaptation sociale, l'étude montre que, comparativement au groupe témoin, une caractéristique toujours déterminante des élèves du groupe TC est leur fréquent recours aux conduites d'agression, particulièrement à l'agression verbale, pour résoudre leurs différends. Mais ce résultat peut aussi refléter le fait qu'encore plus de la moitié des élèves du groupe TC présente un trouble de nature antisociale au troisième cycle du primaire, qu'il s'agisse d'un trouble oppositionnel ou d'un trouble des conduites. Néanmoins, l'adaptation sociale de ces élèves est sensiblement comparable à celle du groupe témoin pour ce qui est de la délinquance et de la consommation de psychotropes, deux importants facteurs d'aggravation et de persistance des troubles de comportement à l'adolescence (Laventure, Déry et Pauzé, 2006b; Moffitt et Caspi, 2006). Il s'agit encore ici d'un résultat encourageant qui peut aussi refléter le rôle positif des services éducatifs complémentaires sur la non-aggravation des troubles des élèves - ceci, dit avec toutes les réserves que nous avons émises précédemment. Il demeure cependant possible que les élèves qui ont participé à l'étude soient trop jeunes pour s'adonner à de telles conduites. Par exemple, dans la population générale, près de $13 \%$ des élèves de $1^{\text {re }}$ secondaire rapportent avoir bu de l'alcool à 9 ans et $2 \%$ avoir fait usage de cannabis à 11 ans (Adlaf, Offord 
et Ogborne, 2000), des taux qui sont relativement proches de ceux rapportés ici. Néanmoins, en observant les résultats systématiquement plus élevés dans le groupe TC que dans le groupe témoin, nous ne pouvons exclure le fait que l'absence de différence significative sur certaines variables soit liée au faible nombre de participants dans l'étude, ce qui contribue à réduire la puissance statistique nécessaire à la détection de différences fines entre les groupes.

En rapportant les résultats de l'étude, nous avons relevé que près de $14 \%$ des élèves du groupe témoin (soit six élèves) avaient aussi reçu des services éducatifs complémentaires pour des difficultés comportementales au cours de l'année scolaire. Ce taux est élevé si on le compare aux 2,5\% attendus selon les chiffres avancés par le Conseil supérieur de l'éducation (2001). Il peut donc s'agir d'un biais de sélection lié au fait que les élèves du groupe témoin aient été recrutés dans des secteurs initialement ciblés pour réduire les écarts socioéconomiques avec le groupe TC. Le groupe témoin pouvant contenir un nombre plus élevé qu'attendu d'élèves avec des difficultés comportementales - ce qui peut réduire les différences entre les groupes -, nous avons refait toutes les analyses se rapportant au deuxième objectif de l'étude en retirant du groupe témoin les six enfants qui avaient reçu des services éducatifs complémentaires. Le retrait de ces élèves a toutefois conduit aux mêmes résultats.

L'interprétation des résultats est aussi limitée par l'absence d'information sur la nature spécifique et l'intensité des interventions offertes aux élèves. En outre, ces services peuvent ne rendre compte que très partiellement de l'évolution des élèves puisque d'autres facteurs non considérés dans l'étude (les facteurs familiaux, par exemple) ont pu jouer un rôle dans cette évolution. Toutefois, le nombre élevé d'élèves qui présentent toujours des troubles de comportement à la fin de l'école primaire est un résultat tangible qui suggère que l'intervention auprès des élèves qui ont des troubles de comportement devrait être plus intensive. Les intervenants devraient particulièrement cibler les conduites agressives des élèves, ce qui pourrait améliorer la qualité de leur insertion sociale et diminuer encore les risques de développer un mode de vie déviant. Le renforcement des compétences en français devrait aussi être ciblé pour éviter l'échec scolaire et bien préparer les élèves à leur passage au secondaire.

De manière générale, la gravité des troubles présentés par les élèves et leur persistance dans le temps questionnent plus fondamentalement la capacité de l'école à assumer la réadaptation de ces enfants. Au terme de cette étude, nous ne pouvons que souhaiter que les collaborations entre le secteur de l'éducation et le secteur de la santé et des services sociaux se multiplient pour soutenir les enseignants et les professionnels non enseignants qui viennent en aide à ces jeunes élèves.

\section{Références}

Achenbach, T.M. et Edelbrock, C. (1983). Manual for the Child Behavior Checklist and Revised Child Profile. New York, NY: Queen City Printers.

Adlaf, E.M., Offord, D.R. et Ogborne, E.C. (2000). Age at first alcohol use : A risk factor for the development of alcohol disorders. The American Journal of Psychiatry, 157(5), 745-751.

Alvarez, H.K. et Ollendick, T.H. (2003). Individual and psychosocial risk factors. In C.A. Essau (dir.), Conduct and oppositional defiant disorders: Epidemiology, risk factors and treatment (p. 97-116). Mahvah, NJ: Lawrence Erlbaum.

American Psychiatric Association (1987). Diagnostic and statistical manual of mental disorders (3e éd.) Washington, DC: APA 
American Psychiatric Association (2000). Diagnostic and statistical manual of mental disorders (4e édition, texte révisé). Washington, DC: APA.

Battin-Pearson, S., Newcomb, M.D., Abbott, R.D., Hill, K.G., Catalano, R.F. et Hawkins, J.D. (2000). Predictors of early high school dropout: A test of five theories. Journal of Educational Psychology, 92(3), 568-582.

Beekhoven, S. et Dekkers, H. (2005). Early school leaving in the lower vocational track: Triangulation of qualitative and quantitative data. Adolescence, 40(157), 197-213.

Bjorkqvist, K., Lagerspetz, K.M.J. et Österman, K. (1992). The direct and indirect aggression scales. Vasa: Abo Akademi University, Department of Social Sciences.

Breton, J.J., Bergeron, L., Valla, J.-P., Berthiaume, C. et St-Georges, M. (1998). The diagnostic interview schedule for children (DISC 2.25) in Quebec. Reliability findings in the light of the MECA study. Journal of American Academy of Child and Adolescent Psychiatry, 37, 1167-1174.

Breton, J.-J., Bergeron, L., Valla, J.-P., Berthiaume, C., Gaudet, N., Lambert, J., St-Georges, M., Houde, L. et Lépine, S. (1999). Quebec child mental health survey: Prevalence of DSM-III-R mental health disorders. Journal of Child Psychology and Psychiatry, 40, 375-384.

Conseil supérieur de l'éducation (2001). Les élèves en difficulté de comportement à l'école primaire: comprendre, prévenir, intervenir. Québec: CSÉ.

Déry, M., Toupin, J., Pauzé, R. et Verlaan, P. (2004). Frequency of mental health disorders in a sample of elementary school students receiving special educational services for behavioral difficulties. Canadian Journal of Psychiatry, 49, 175-181.

Déry, M., Toupin, J., Pauzé, R. et Verlaan, P. (2005). Les caractéristiques d'élèves en difficulté de comportement placés en classe spéciale ou intégrés dans la classe ordinaire. Revue canadienne de l'éducation, 28(1/2), 1-23.

Dunn, L.M., Thériault-Whalen, C.M. et Dunn, L.M. (1993). Échelle de vocabulaire en images Peabody. Toronto: Psychan.

Fortin, L., Royer, É., Potvin, P., Marcotte, D. et Yergeau, É. (2004). La prédiction du risque du décrochage scolaire au secondaire: facteurs personnels, familiaux et scolaires. Revue canadienne des sciences du comportement, 36(3), 219-231.

Fortin, L. et Strayer, F.F. (2000). Caractéristiques de l'élève en troubles du comportement et contraintes sociales du contexte. Revue des sciences de l'éducation, XXVI(1), 3-16.

Frick, P.J. (2004). Developmental pathways to conduct disorder: Implications for serving youth who show severe aggressive and antisocial behavior. Psychology in the School, 41(8), 823-834.

Gagnon, C. et Vitaro, F. (2003). La prévention du trouble des conduites avec centration sur les comportements violents. In F. Vitaro et C. Gagnon (dir.), Prévention des problèmes d'adaptation chez les enfants et les adolescents. Tome II. Les problèmes externalisés. (p. 231-290). Québec: Presses de l’Université du Québec.

Germain, M., Guyon, L., Landry, M., Tremblay, J., Brunelle, N. et Bergeron, J. (2005). Grille de dépistage de consommation problématique d'alcool et de drogues chez les adolescents et les adolescentes - DEP-ADO. Version 3.1. Montréal: Recherche et intervention sur les substances psychoactives - Québec (RISQ).

Gouvernement du Québec (1999). Une école adaptée à tous ses élèves. Politique de l'adaptation scolaire. Québec: Ministère de l'Éducation.

Gouvernement du Québec (2002). Les services éducatifs complémentaires: essentiels à la réussite. Québec: Ministère de l'Éducation.

Hinshaw, S.P. et Lee., S.S. (2003). Conduct and oppositional defiant disorders. In E.J. Mash et R.A. Barkley (dir.), Child psychopathology (p. 144-198). New York, NY: Guilford Press.

Jensen, P., Rubio-Stipec, M., Canino, G., Bird, H., Dulcan, M. et Schwab-Stone, M.E. (1999). Parent and child contributions to diagnosis of mental disorder: Are both informants always necessary? Journal of American Academy of Child and Adolescent Psychiatry, 38, 1569-1579.

Kazdin, A.E. et Wassell, G. (2000). Therapeutic changes in children, parents and families resulting form treatment of children with conduct problems. Journal of the American Academy of Child and Adolescent Psychiatry, 39(4), 414-420. 
Kershaw, P. et Sonuga-Barke, E. (1998). Emotional and behavioural difficulties: Is this a useful category? The implications of clustering and co-morbidity, the relevance of a taxonomic approach. Educational and Child Psychology, 15, 45-55.

Kuperman, S., Chan, G., Kramer, J.R. et Bierut, L. (2005). Relationship of age first drink to child behavioral problem and family psychopathology. Alcoholism: Clinical and Experimental Research, 29(10), 1869-1876.

Lahey, B.B., Schwab-Stone, M., Goodman, S.H., Waldman, I.D., Canino, G., Rathouz, P.J., Miller, T.L., Dennis, K.D., Bird, H. et Jensen, P.S. (2000). Age and gender differences in oppositional behavior and conduct problems: A cross-sectional household study of middle childhood and adolescence. Journal of Abnormal Psychology, 109(3), 488-503.

Lambert, N. (2005). The contribution of childhood ADHD, conduct problems, and stimulant treatment to adolescent and adult tobacco and psychoactive substance abuse. Ethical Human Psychology and Psychiatry, 7(3), 197-221.

Laventure, M., Déry, M. et Pauzé, R. (2006a). Sévérité de la consommation de psychotropes des jeunes ayant un trouble des conduites. Revue de criminologie, 39(2), 165-188.

Laventure, M., Déry, M. et Pauzé, R. (2006b). Consommation de psychotropes et persistance du trouble des conduites. Alcoologie et addictologie, 28(3), 223-230.

Loeber, R. et Keenan, K. (1994). Interaction between conduct disorder and its comorbid conditions : Effects of age and gender. Clinical Psychology Review, 14(6), 497-523.

Lynam, D., Moffitt, T. et Stouthamer-Loeber, M. (1993). Explaining the relation between IQ and delinquency: class, race, test motivation, school failure, or self-control? Journal of Abnormal Psychology, 102, 187-196.

Mattison, R.E., Gadow, K.D., Sprafkin, J. et Nolan, E.E. (2002). Discriminant validity of a DSM-IV-based teacher checklist: Comparison of regular and special education students. Behavioral Disorders, 27, 304-316.

Menard, S. (2001). Applied logistic regression analysis. Sage university papers series on quantitative applications in the social sciences, series no. 07-106. Thousand Oaks, CA: Sage Publications.

Mitsis, E.M., McKay, K.E., Schulz, K.P., Newcorn, J.H. et Halperin, J.M. (2000). Parent-teacher concordance for DSM-IV Attention-Deficit/Hyperactivity Disorder in a clinic-referred sample. Journal of American Academy of Child and Adolescent Psychiatry, 39, 308-313.

Moffitt, T.E. et Caspi, A. (2006). Facteurs de risque associés aux trajectoires développementales des conduites antisociales chez les garçons et les filles. In P. Verlaan et M. Déry (dir.), Les conduites antisociales chez les filles: Comprendre pour mieux agir (p. 79-119). Québec: Presses de l'Université du Québec.

Muthén, B. et Curran, P. (1997). General longitudinal modeling of individual differences in experimental designs: A latent variable framework for analysis and power estimation. Psychological Methods, 2, 371-402.

Nelson, J.R., Babyak, A., Gonzalez, J. et Benner, G.J. (2003). An investigation of problem behaviors exhibited by K-12 students with emotional or behavioral disorders in public school settings. Behavioral Disorders, 28, 348-359.

Place, M., Wilson, J., Martin, E. et Hulsmeier, L. (2000). The frequency of emotional and behavioural disturbance in an EBD school. Child Psychology and Psychiatry Review, 5, 76-80.

Statistique Canada (1996). Enquête longitudinale nationale sur les enfants et les jeunes. Matériel d'enquête pour la collecte des données. Hull: Développement des ressources humaines Canada. Catalogue no. 89FOO77XPF.

Toupin, J. (1993). Échelle de statut socioéconomique. Sherbrooke: Université de Sherbrooke, Faculté d'éducation, Groupe de recherche sur les inadaptations sociales de l'enfance (GRISE).

Toupin, J., Déry, M., Pauzé, R., Mercier, H. et Fortin, L. (2000). Social and cognitive contributions to conduct disorder in children. Journal of Child Psychology and Psychiatry, 41, 333-44.

Trépanier, N. et Dessureault, D. (2006). Les principes et le cadre d'intervention auprès des élèves ayant des difficultés de comportement. In L. Massé, N. Desbiens et C. Lanaris (dir.), Les troubles du comportement à l'école. Prévention, évaluation et intervention (p. 85-102). Montréal: Gaëtan Morin.

Valla, J.P., Breton, J.J., Bergeron, L., Gaudet, N., Berthiaume, C., St-Georges, M., Daveluy, C., Tremblay, V., Lambert, J., Houde, L. et Lépine, S. (1994). Enquête québécoise sur la santé mentale des jeunes de 6 à 14 ans. Rapport synthèse. Montréal: Hôpital Rivières-des-Prairies et Santé Québec. 
Vernberg, E.M., Roberts, M.C., Randall, C.J., Biggs, B.K., Nyre, J.E. et Jacobs, A.K. (2006). Intensive mental health services for children with serious emotional disturbances through a school-based, community-oriented program. Clinical Child Psychology and Psychiatry, 11(3), 417-430.

Vitaro, F., Brendgen, M., Pagani, L., Tremblay, R.E., McDuff, P. (1999). Disruptive behavior, peer association, and conduct disorder: Testing the developmental link through early intervention. Development and Psychopathology, 11, 287-304.

Waschbusch, D.A. (2002). A meta-analytic examination of comorbid hyperactive-impulsive-attention problems and conduct problems. Psychological Bulletin, 128(1), 118-150.

Williams, R.J. et Nowatzki, N. (2005). Validity of adolescent self-report of substance use. Substance Use and Misuse, 40, 299-311. 